\title{
Do plant volatiles confuse rather than guide foraging behavior of the aphid hyperparasitoid Dendrocerus aphidum?
}

\author{
Jetske G. de Boer ${ }^{1}\left[\right.$ ] Petra J. Hollander ${ }^{1} \cdot$ Daan Heinen $^{1} \cdot$ Divya Jagger $^{1} \cdot$ Pim van Sliedregt $^{1} \cdot$ Lucia Salis $^{1}$. \\ Martine Kos $^{2}$ (D) Louise E. M. Vet ${ }^{1,2}$ (1)
}

Received: 20 May 2020 / Accepted: 29 July 2020 / Published online: 25 August 2020

(c) The Author(s) 2020

\begin{abstract}
Many species of parasitoid wasps use plant volatiles to locate their herbivorous hosts. These volatiles are reliable indicators of host presence when their emission in plants is induced by herbivory. Hyperparasitoids may also use information from lower trophic levels to locate their parasitoid hosts but little is known about the role of volatiles from the plant-host complex in the foraging behavior of hyperparasitoids. Here, we studied how Dendrocerus aphidum (Megaspilidae) responds to plant and host volatiles in a series of experiments. This hyperparasitoid uses aphid mummies as its host and hampers biological control of aphids by parasitoids in greenhouse horticulture. We found that $D$. aphidum females were strongly attracted to volatiles from mummy-infested sweet pepper plants, but only when clean air was offered as an alternative odor source in the Y-tube olfactometer. Hyperparasitoid females did not have a preference for mummy-infested plants when volatiles from aphid-infested or healthy pepper plants were presented as an alternative. These olfactory responses of D. aphidum were mostly independent of prior experience. Volatiles from the host itself were also highly attractive to $D$. aphidum, but again hyperparasitoid females only had a preference in the absence of plant volatiles. Our findings suggest that plant volatiles may confuse, rather than guide the foraging behavior of $D$. aphidum. Mummy hyperparasitoids, such as $D$. aphidum, can use a wide variety of mummies and are thus extreme generalists at the lower trophic levels, which may explain the limited role of (induced) plant volatiles in their host searching behavior.
\end{abstract}

Keywords Associative learning $\cdot$ Fourth trophic level $\cdot$ Herbivore-induced plant volatiles $\cdot$ Host searching $\cdot$ Infochemicals . Microbial volatiles

\section{Introduction}

It is well established that parasitoid wasps are attracted to plant volatiles. In particular, plants emit specific blends of volatiles after induction by herbivory (McCormick et al. 2012). To a foraging parasitoid, these so-called

Communicated by Günther Raspotnig.

Electronic supplementary material The online version of this article (https://doi.org/10.1007/s00049-020-00321-5) contains supplementary material, which is available to authorized users.

Jetske G. de Boer

j.deboer@nioo.knaw.nl

1 Department of Terrestrial Ecology, Netherlands Institute of Ecology (NIOO-KNAW), Wageningen, The Netherlands

2 Laboratory of Entomology, Wageningen University, Wageningen, The Netherlands herbivore-induced plant volatiles (HIPVs) can convey reliable information about the specific herbivore that is feeding on the plant, and HIPVs are more detectable than volatiles from the herbivorous host itself (Vet et al. 1991). The ecological and evolutionary implications of infochemicals from the first and the second trophic level for parasitoid foraging behavior were first described by Vet and Dicke (1992). By attracting the natural enemies of their herbivorous attackers, feeding damage on plants may be reduced, leading to better performance of plants that emit HIPVs. Hence, HIPVs shape multitrophic interactions in food-webs and are of evolutionary significance (Vet 1999; Dicke and Van Loon 2000). HIPVs have been studied extensively in a tritrophic context, advancing knowledge on the mechanisms of induction and production, and on the role of HIPVs in foraging behavior of parasitoids (Turlings and Erb 2018). Other organisms in the environment may also perceive and respond to infochemicals emitted by plants, and (induced) plant volatiles may, 
therefore, mediate interactions beyond the third trophic level (Poelman and Kos 2016). In this respect, hyperparasitoids (the fourth trophic level) are particularly interesting because they are natural enemies of parasitoid wasps. In horti- and agriculture, hyperparasitoids are considered problematic because they kill primary parasitoids that are often used as biological control agents of pest insects, and can prevent establishment of primary parasitoids (Sullivan and Völkl 1999). Besides being of fundamental interest, knowledge on the role of volatiles in foraging behavior of hyperparasitoids may also contribute to managing them in agriculture (Cusumano et al. 2020). We address this topic here by studying olfactory responses of the aphid hyperparasitoid Dendrocerus aphidum Rondani (Megaspilidae) to plant and host volatiles.

To date, the role of plant volatiles in foraging behavior of fourth trophic level hyperparasitoids is not well understood (Cusumano et al. 2020), with few exceptions. Lysibia nana is a hyperparasitoid of cocoons of Cotesia glomerata, which parasitizes caterpillars of the cabbage white butterfly. This hyperparasitoid prefers volatiles from plants infested with parasitized caterpillars to volatiles from plants damaged by healthy caterpillars (Poelman et al. 2012; Zhu et al. 2015). In this system, parasitism status of the caterpillar plays a more important role in attraction of L. nana than herbivore identity. Blends of HIPVs indeed differ according to parasitism status (Poelman et al. 2012) and this effect is mediated by symbiotic polydna viruses that are transferred to the caterpillar during parasitism and affect the plant through the caterpillar's saliva (Zhu et al. 2018). Laboratory and field experiments further showed that $L$. nana can use HIPVs to distinguish between plants with caterpillars parasitized by the non-host Hyposoter ebeninus and its host $C$. glomerata (Cusumano et al. 2019). A few hyperparasitoid species associated with aphids are also known to respond to plant volatiles, but these studies were not aimed at specific induction of volatiles by (parasitized) aphids (Singh and Srivastava 1987a, b; Siri 1993; Völkl and Sullivan 2000). Together, these studies show that (induced) plant volatiles can have consequences beyond the third trophic level, warranting further research on when, why and how plant volatiles play a role in hyperparasitoid functioning.

Compared to plant volatiles, volatiles from the second or third trophic level may be more reliable to foraging hyperparasitoids, in particular when these two trophic levels interact. Indeed, Baryscapus galactopus is more attracted to parasitized caterpillars than to healthy caterpillars, and this preference can be explained by changes in the body odor profile of parasitized caterpillars (Zhu et al. 2014). Similarly, Alloxysta victrix is arrested by the smell of parasitized aphids compared to the smell of healthy aphids (Grasswitz 1998), and it was suggested that Dendrocerus carpenteri is attracted to aphid mummies, which are its host (Siri 1993).
However, results for aphid hyperparasitoids vary because these findings were not supported by a later study on foraging behavior of four species, including $A$. victrix and $D$. carpenteri (Buitenhuis et al. 2005). Instead, Buitenhuis et al. (2005) showed that three hyperparasitoid species were influenced by aphid honeydew, which acted as a search stimulant on the plant. In this case, there does not seem to be an interaction between the second and third trophic level because aphid hyperparasitoids do not respond differently to honeydew from healthy or parasitized aphids (Buitenhuis et al. 2004). Moreover, after parasitized aphids turn into mummies and stop feeding on the plant, they no longer produce honeydew. Instead of directing hyperparasitoids to their host, attraction to honeydew may be driven by the substantial impact of this carbohydrate-rich food source on longevity and fecundity of aphid hyperparasitoids (van Neerbos et al. 2020).

Here, we studied the aphid mummy hyperparasitoid Dendrocerus aphidum. Like most Dendrocerus species, $D$. aphidum parasitizes its host in the mummy stage (the aphid remains containing the primary parasitoid) by laying a single egg under the mummy skin. The larva feeds on the primary parasitoid (pre)pupa and emerges as an adult approximately two weeks later. Adult females feed on floral nectar or aphid honeydew, which extends their lifespan substantially (de Boer et al. 2019). Dendrocerus aphidum commonly occurs in Dutch sweet pepper greenhouses, where it can disrupt biological control of aphids (Bloemhard et al. 2014; de Boer et al. 2019). We, therefore, used a study system consisting of sweet pepper plants (Capsicum annuиm), the tobacco aphid Myzus persicae nicotianae and its main parasitoid Aphidius colemani (Braconidae), which is often used as a commercial biocontrol agent of $M$. persicae. We investigated the relative importance of plant and host volatiles in foraging behavior of $D$. aphidum by:

(1) Evaluating the olfactory response of D. aphidum females towards volatiles derived from plant-insect complexes (i.e. plants infested with mummies and/or (parasitized) aphids and uninfested plants).

(2) Determining the effect of plant volatiles on attraction of $D$. aphidum to host volatiles.

We expected $D$. aphidum to respond most strongly to plants infested with mummies because these plants contain suitable hosts and present the complete suite of infochemicals. Uninfested plants or plants with (parasitized) aphids contain no hosts (yet) and, therefore, present less reliable information to D. aphidum. It is well known that prior exposure to the plant-host complex can influence olfactory responses of primary parasitoids, in particular when HIPVs are combined with an oviposition experience (Vet et al. 1990; Kruidhof et al. 2019; Little et al. 2019). We, 
therefore, compared the responses of naïve and experienced hyperparasitoids, expecting a stronger attraction of experienced females to plant volatiles.

\section{Methods}

\section{Plants and insects}

Sweet pepper plants (Capsicum annuum var. Maranello) and winter wheat plants (Triticum aestivum var. Premio) were grown from seeds in a greenhouse using conditions and methods described in van Neerbos et al. (2020). Pepper plants were grown individually in pots $(1.45 \mathrm{~L}, 13 \mathrm{~cm}$ diameter) and used in experiments 5-6 weeks after sowing. Wheat plants were grown in pots with a surface of $25 \mathrm{~cm}^{2}$ at a density of approximately 1 plant per $\mathrm{cm}^{2}$, and used 1 week after sowing. Myzus persicae nicotianae and Rhopalosiphum padi aphids were cultured on pepper and wheat plants, respectively, in large mesh cages $(60 \times 60 \times 60 \mathrm{~cm}$, Bugdorm) in climate-controlled chambers. To maintain colonies, fresh plants were added to these cages three times per week. Aphidius colemani were obtained from Koppert Biological Systems (Berkel en Rodenrijs, The Netherlands) as mummies and used to parasitize M. persicae and R. padi aphids after emergence. Pepper plants with A. colemani mummies developed on $M$. persicae were used to maintain a colony of the aphid hyperparasitoid Dendrocerus aphi$d u m$. This colony originated from adults and hyperparasitized mummies collected in a sweet pepper greenhouse in Schalkwijk (The Netherlands) in 2018 and they were identified to species by F. van Veen (University of Exeter) and the first author. Hyperparasitoids were kept in fine mesh cages $(30 \times 30 \times 30 \mathrm{~cm}$, Bugdorm $)$ and provided with water, honey and fresh aphid mummies on clipped pepper plants several times per week as described in de Boer et al. (2019). Unless mentioned otherwise, in experiments, we used, presumably mated, female $D$. aphidum 2-7 days after emergence. Before testing, they were kept with water and honey but without host mummies (further referred to as naïve females). All plants and insects were kept at $22{ }^{\circ} \mathrm{C}, 50-70 \%$ R.H. and a light:dark cycle of $16 \mathrm{~h}: 8 \mathrm{~h}$.

\section{Y-tube olfactometer}

All olfactory responses were evaluated in a glass Y-tube olfactometer described by Fatouros et al. (2012). In short, carbon-filtered and humidified air was blown through two glass jars $(30 \mathrm{~L})$ connected to the arms of the olfactometer (14 cm in length, $1 \mathrm{~cm}$ diameter). Air-speed was equal in both arms of the olfactometer at 150-250 $\mathrm{ml} / \mathrm{min}$. PTFEtubing was used to connect the glass parts of the system. The Y-tube olfactometer was positioned at a $20^{\circ}$ upward angle and two T5 incandescence lights were placed above the olfactometer to increase the hyperparasitoids' response rate by positive phototaxis and negative geotaxis. A curtain was placed around the observer to block light or other visual stimuli from outside the experimental setup. Experiments started approximately one hour after placing the odor sources in the glass jars. In experiments with plants, their pots were wrapped in tin foil to minimize interference with odors from the soil. In single-choice experiments with only one glass jar holding a plant, a white paper was placed around both glass jars to exclude visual stimuli from plants. This was not done in other experiments because we assumed that visual stimuli were symmetrical in those cases. All experiments were done at room temperature $\left(20 \pm 2{ }^{\circ} \mathrm{C}\right)$.

Hyperparasitoid females were introduced individually into the olfactometer, using a glass tube that fitted into the opening of the Y-tube olfactometer, or a syringe that was cut open. Every female was given a maximum of $10 \mathrm{~min}$ to choose between the two odor sources and a choice was marked when the female passed a line approximately $8 \mathrm{~cm}$ up one arm of the olfactometer. If an individual exceeded the given time, it was marked as no response. New odor sources were prepared for each experimental day. Per day, the odor sources were tested on both sides of the olfactometer by switching the tubes that connected the jars with the arms of the Y-tube to minimize any effects of a positional bias. Unless mentioned otherwise, only one treatment was tested per day to avoid an effect of odor of one treatment affecting another test. Glass jars, Y-tube and PTFE-tubes were cleaned with $70 \%$ ethanol and placed in an oven at $105{ }^{\circ} \mathrm{C}$ for two hours to minimize effects of odor residues between experimental days.

\section{Experiment 1: olfactory response to plant-insect complexes versus clean air}

We first investigated the olfactory response of $D$. aphidum to volatiles from the entire plant-host complex (infested with mummies), plants infested with (parasitized) aphids, and uninfested plants against clean air. To obtain mummyinfested plants (M-plants), we selected 5-6-week-old vegetative sweet pepper plants and infested them with 50 adult $M$. persicae aphids 11 days before the experiment. Adult aphids were removed after $24 \mathrm{~h}$ and their nymphs were parasitized by $A$. colemani 2 days later. Fifty parasitoids were used per plant and they were kept with the plant for $8 \mathrm{~h}$. Plants were then kept for another 8 days to allow development of parasitoids and mummification of aphids. This approach resulted in plants infested with approximately 100 aphids and a parasitism rate of approximately $70 \%$. M-plants, therefore, contained unparasitized aphids and aphid mummies, and possibly some aphids that were parasitized but had not yet turned into mummies. Fully developed mummies were counted 
after testing the plant in the Y-tube olfactometer. Plants with parasitized aphids (PA-plants) were prepared in the same way, and tested 5 days after parasitism (i.e. 8 days after placing aphids on the plants). Parasitism rates on PA-plants were determined by counting mummies and unparasitized aphids three days after the experiments. Aphid-infested plants (A-plants) were prepared 8 days before experiments, allowing nymphs to reach the adult stage. To determine whether prior experience with the plant-host complex affected their olfactory response, we tested two groups of D. aphidum females. Naïve females had not been exposed to host mummies after emergence, while experienced females were offered A. colemani mummies on an infested sweet pepper leaf for $24 \mathrm{~h}$ and were thus provided hosts in which they could oviposit along with the infochemicals of the plant-host complex. After this exposure, they were kept without hosts for another $24 \mathrm{~h}$ before using them in the Y-tube olfactometer. On some experimental days, naïve and experienced females were tested with the same odor source, on other experimental days either naïve or experienced females were tested. In total, 53-84 D. aphidum females were tested per combination of hyperparasitoid and plant treatment, using five or six individual plants per combination.

\section{Experiment 2: olfactory response to mummy-infested plants versus other plant volatiles}

In the first experiment, we established that volatiles from the intact plant-host complex were attractive to naïve and experienced $D$. aphidum females when tested against clean air. Because this is not a realistic situation in the field for foraging hyperparasitoids, we used a more realistic alternative odor source consisting of plant volatiles in the next series of experiments. Three sub-experiments were done:

(I) Volatiles from mummy-infested sweet pepper plants were tested against volatiles from aphid-infested plants, preparing plants as described above. We hypothesized that $D$. aphidum would prefer volatiles from M-plants over those from A-plants. A total of 71 naïve and 71 experienced $D$. aphidum females (as described above) were tested over five experimental days, with both groups of hyperparasitoids tested on the same days.

(II) Volatiles from mummy-infested sweet pepper plants were tested against uninfested plants. Due to low numbers of mummies on M-plants compared to the experiments described above, we made some adjustments in the preparation of M-plants by varying the age of aphids when they were parasitized and changing exposure of aphids to parasitoids (see supplementary information for details and results).
Because numbers of mummies were variable and on average lower than in previous experiments, and because the proportion of hyperparasitoids that made a choice was low overall, this experiment was performed with a larger number of individual plants. We found no effect of the number of mummies on the response of hyperparasitoids and, therefore, data from all replicates are presented in the results section. In total, 200 naïve and 203 experienced $D$. aphidum females were tested, using 10 and 17 individual plants, respectively, with naïve and experienced hyperparasitoids tested on different days.

(III) To evaluate if our results were influenced by the plant species we used, we repeated sub-experiment 2-II with wheat. Wheat plants and $R$. padi aphids are likely more representative of the food-web in which Dendrocerus hyperparasitoids evolved than pepper plants infested with M. persicae (Fergusson 1980). Volatiles from mummy-infested wheat plants were tested against uninfested wheat plants. Mummy-infested wheat plants were obtained by placing pots with wheat plants next to wheat plants infested with $R$. padi aphids in a large mesh cage for $24 \mathrm{~h}$. Infested plants were then transferred to another large mesh cage with newly emerged $A$. colemani parasitoids for $24 \mathrm{~h}$. Plants with parasitized aphids were then kept in a mesh cage for one week until mummies formed. Uninfested wheat plants were kept in a mesh cage under the same conditions. Numbers of aphids and parasitoids were not controlled precisely because it is difficult to transfer aphids to and remove them from wheat plants because of the vertical position of the leaves. The number of mummies was determined after plants were used in the Y-tube olfactometer (Supplementary Fig. S1). Three hyperparasitoid treatments were used: naïve females, and females exposed for $24 \mathrm{~h}$ to a mummy-infested pepper leaf (as described above, pepper-experienced) or to mummy-infested wheat plants (wheat-experienced). Per treatment, 56-61 D. aphidum females were tested, using 8-10 individual pots of mummyinfested wheat plants. Naïve and experienced hyperparasitoids were sometimes tested on the same day with the same wheat plants but not on all experimental days.

\section{Experiment 3: attractiveness of mummy volatiles in the absence or presence of plant volatiles}

In the first two experiments, we did not remove insects (aphids and mummies) from infested plants, and odor 
sources thus comprised mummy and aphid volatiles as well as (induced) plant volatiles. Because this approach did not allow us to assess the importance of (induced) plant volatiles and host volatiles, we separated host and plant volatiles in the last experiment. Per experimental day, we investigated the olfactory response of $D$. aphidum to mummy volatiles and then added a background of plant volatiles from uninfested sweet pepper plants. Mummies were obtained from our colony of A. colemani on M. persicae on sweet pepper by collecting mummies with a fine paintbrush approximately $24 \mathrm{~h}$ after mummy formation. A set of 100 mummies was enclosed in the PTFE-tube that connected one of the glass jars to the Y-tube olfactometer. A small piece of nylon mesh was wrapped around the end of the tube and tightened with PTFE tape to prevent mummies from falling out of the tube. A similar piece of nylon mesh and PTFE tape were wrapped around the PTFE air outlet of the second glass jar as a control. Per experimental day, we first tested the response of 9-14 naïve $D$. aphidum to volatiles from $100 \mathrm{~A}$. colemani mummies in the absence of plant volatiles. Then, a 6-week-old sweet pepper plant was placed in each of the glass jars. After $15 \mathrm{~min}$, we tested the response of another set of 11-14 naïve $D$. aphidum females to mummy volatiles in the presence of plant volatiles. These tests were repeated on 6 experimental days.

\section{Statistical analyses}

Statistical analyses were performed in R (version 3.5.0). In generalized linear models (GLM), the number of hyperparasitoids attracted to an individual (infested) plant was used as the response variable and the total number of hyperparasitoids that made a choice was used as the binomial total. For the model on the proportion of responding hyperparasitoids, the total number of hyperparasitoids that made a choice was used as the response variable and the total number tested was used as the binomial total. A binomial distribution was assumed and a logit-link function was used. ANOVAs (with the likelihood ratio test) were used to compare full and reduced models.

In the first experiment, where (infested) pepper plants were tested against clean air, we first used an overall GLM with $D$. aphidum treatment (naïve versus experienced) and plant treatment (uninfested, aphid-infested, parasitized aphid-infested or mummy-infested) and their interaction as explanatory factors. Next, to determine if plants of the different treatments attracted D. aphidum, we ran separate models per plant treatment with hyperparasitoid treatment as an explanatory factor. Choice of $D$. aphidum between the treatment and control odor sources was statistically compared to a random 50:50 distribution by testing if the intercept of the model was significantly different from 0 .
This was done per plant treatment, including hyperparasitoid treatment in the model when it was significant.

The three sub-experiments with volatiles from mummyinfested plants as an odor source were analyzed separately. For each, we used a GLM with hyperparasitoid treatment (naïve versus experienced) and the number of mummies on the mummy-infested plant as explanatory variables. In the experiment with infested wheat plants, hyperparasitoid treatment included three levels: naïve, wheat-experienced and pepper-experienced. Choice of D. aphidum was compared against a random 50:50 distribution as described above.

In the experiments with mummies as an odor source, the effect of adding plants to mummies was tested in a GLM with 'plant' as an explanatory factor. We also evaluated the effect of 'plant' on the proportion of responding hyperparasitoids in a GLM.

\section{Results}

\section{Experiment 1: olfactory response to plant-insect complexes versus clean air}

We found a significant interaction between plant treatment and hyperparasitoid treatment on the olfactory response of D. aphidum females to volatiles from the plant(-host) complex in the Y-tube olfactometer (GLM, $\chi^{2}{ }_{3}=8.23, P=0.041$; Fig. 1). This suggests that the effect of prior exposure to the plant-host complex (naïve versus experienced) on hyperparasitoid choice depended on the odor source offered in the olfactometer (sweet pepper plants with either mummies, parasitized aphids or healthy aphids, or left uninfested). Indeed, hyperparasitoid experience only significantly influenced the response of $D$. aphidum to volatiles from plants infested with parasitized aphids (GLM, $\chi^{2}{ }_{1}=8.93, P=0.003$ ). For the other plant-treatments, we found no significant effect of hyperparasitoid experience. Volatiles from mummy-infested plants were highly attractive to $D$. aphidum (GLM, $z=4.59$, $P<0.001$ ), irrespective of experience, although there was a tendency for experienced hyperparasitoids to be even more attracted than naïve ones (GLM, $\left.\chi^{2}{ }_{1}=3.42, P=0.064\right)$. Volatiles from plants with parasitized aphids were also attractive to hyperparasitoid females (GLM, $z=2.79, P=0.005$ ), with naïve females significantly less attracted than experienced ones (GLM, $\chi^{2}{ }_{1}=8.93, P=0.003$ ). Volatiles from aphidinfested plants, on the other hand, significantly attracted $D$. aphidum (GLM, $z=2.03, P=0.043$ ), independent of experience (GLM, $\chi^{2}{ }_{1}=1.79, P=0.18$ ). Finally, volatiles from uninfested plants did not attract $D$. aphidum females (GLM, $z=-0.10, P=0.92)$ and there was no effect of experience on this response (GLM, $\chi^{2}{ }_{1}=0.75, P=0.39$ ). 


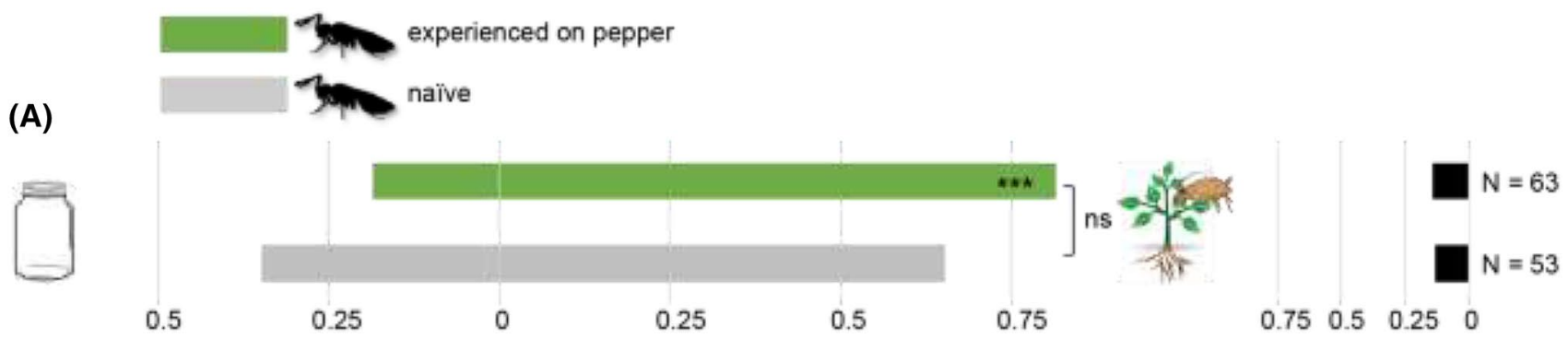

(B)
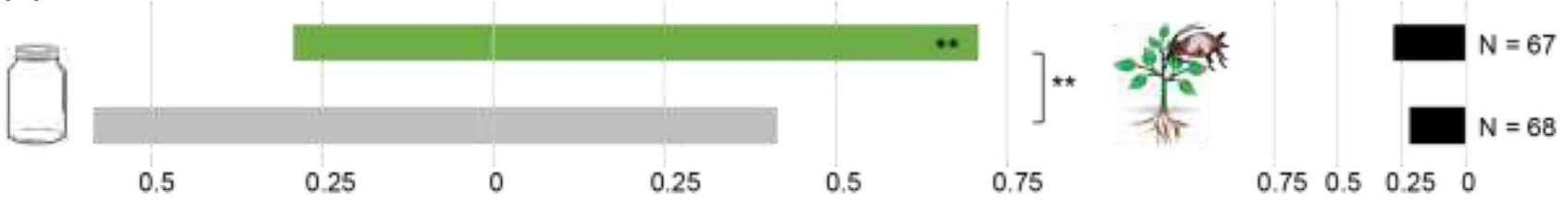

(C)
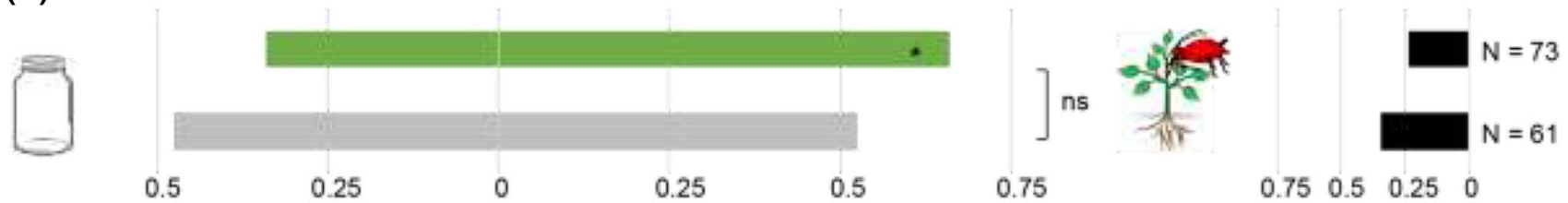

(D)
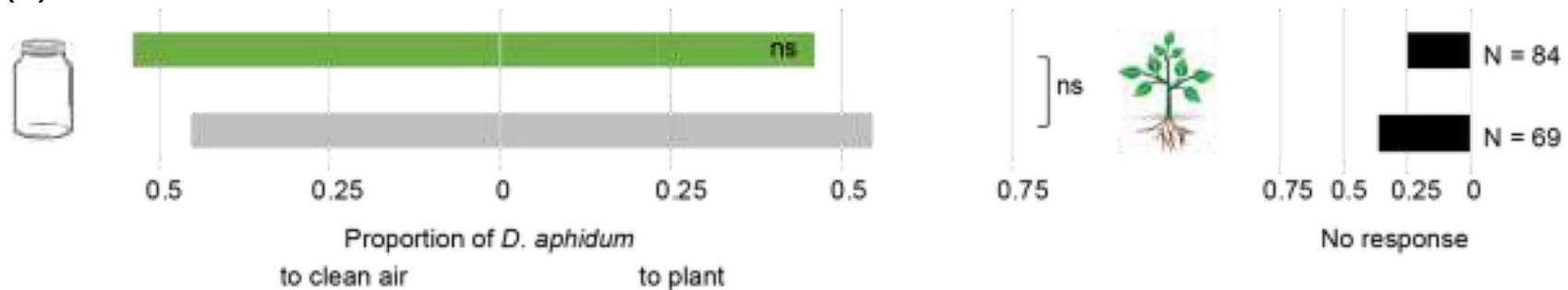

Fig. 1 Response of D. aphidum females to volatiles from (infested) sweet pepper plants in a Y-tube olfactometer. Sweet pepper plants were either infested with mummified (a), parasitized (b) or healthy (c) M. persicae aphids, or left uninfested (d). Bars represent proportions of experienced (green) or naïve (grey) hyperparasitoids that were attracted to the treatment or control odor sources out of the number of hyperparasitoids that made a choice. The proportion of hyperparasitoids that did not respond to either odor source (no response) is presented in the right panels; $\mathrm{N}$ is the total number of individual females tested per combination of hyperparasitoid and

plant treatment, using five or six individual plants per combination. The effect of hyperparasitoid treatment (naïve versus experience) was evaluated per plant treatment (GLM), with significance indicated on the right side of each set of bars. Using the models with significant parameters only, the $P$-value of the intercept was then used to determine whether the choice of $D$. aphidum deviated significantly from a 50:50 distribution (indicated in the green bars because experienced hyperparasitoids were used as the reference level, $P<0.001$, $* * * P<0.01, * * P<0.05, * P>0.05, \mathrm{~ns})$

\section{Experiment 2: olfactory response to mummy-infested plants versus other plant volatiles}

After establishing that volatiles from the plant-host complex (mummy-infested plants) were highly attractive to D. aphidum when tested against clean air, we evaluated hyperparasitoid olfactory response with plant volatiles as an alternative instead of clean air (Fig. 2a-b). Because we did not remove insects (aphids and mummies) from infested plants, odor sources consisting of mummy-infested

plants comprised mummy and aphid volatiles as well as (induced) plant volatiles. Based on our findings in the first experiment, we hypothesized that volatiles from the complete plant-host complex would be preferred by $D$. aphidum. However, hyperparasitoid females did not differentiate between volatiles from mummy-infested and aphidinfested sweet pepper plants (GLM, $z=0.49, P=0.63$ ), or between mummy-infested and uninfested pepper plants (GLM, $z=1.40, P=0.16$ ). In both experiments, this result did not depend on prior exposure of $D$. aphidum females to the plant-host complex (GLM, $\chi^{2}{ }_{1}=0.77, P=0.38$ and 


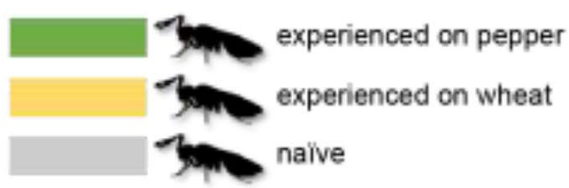

(A)
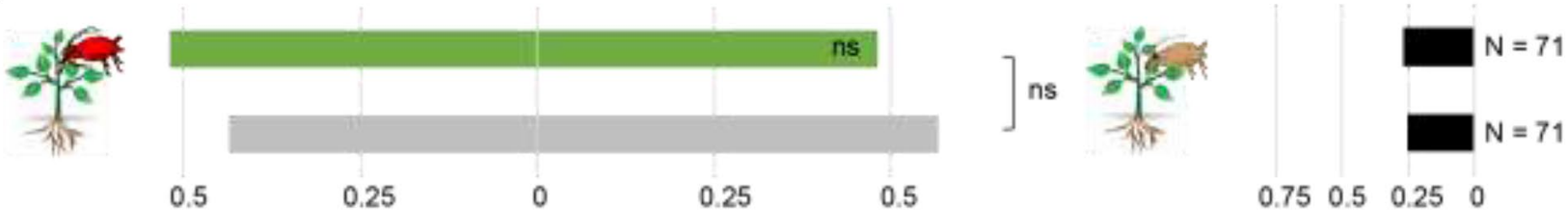

(B)
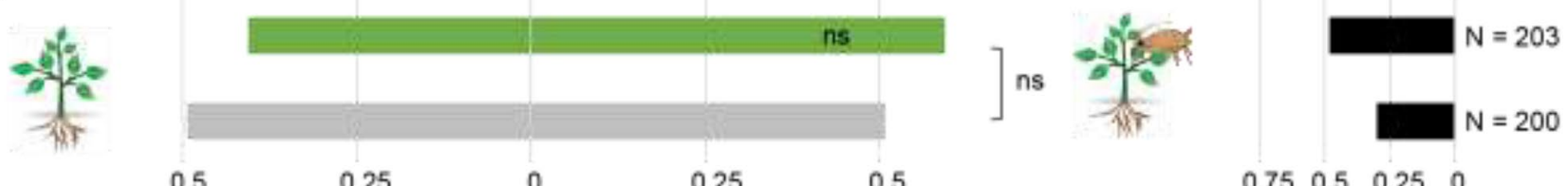

0.5

0.25

0.25

0.75

$\begin{array}{lll}5 & 0.25 & 0\end{array}$

(C)

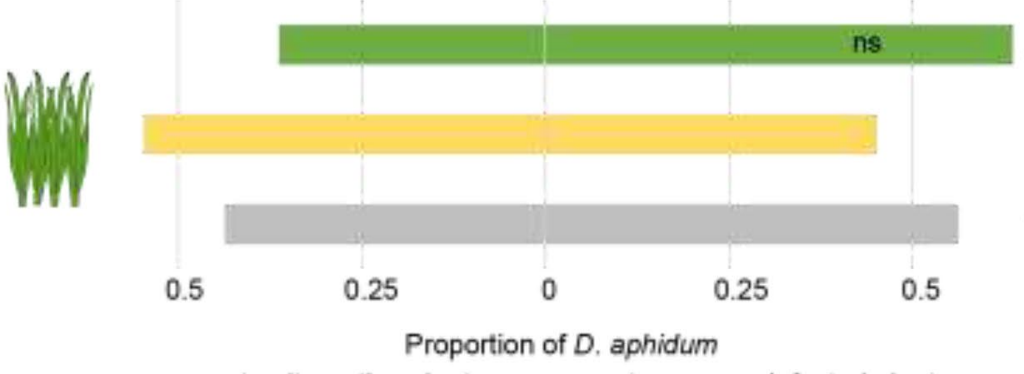

to alternative plant

to mummy-infested plant
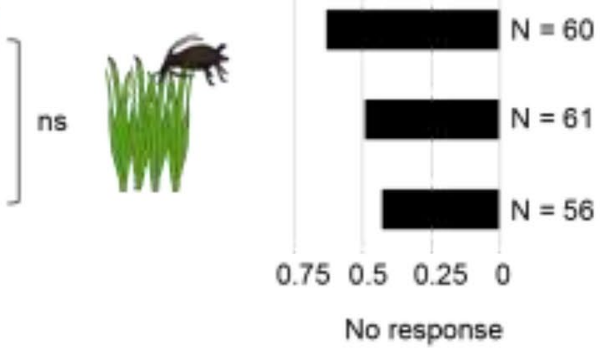

Fig. 2 Response of D. aphidum to volatiles from mummy-infested plants in a Y-tube olfactometer. Hyperparasitoids were offered a choice between sweet pepper plants infested with mummified $M$. persicae aphids and plants infested with healthy aphids (a), or uninfested plants (b), or a choice between wheat plants infested with mummified $R$. padi aphids and uninfested wheat plants (c). Bars represent proportions of naïve (grey) or experienced (green) hyperparasitoids that were attracted to the treatment (mummy-infested) or control odor sources out of the number of hyperparasitoids that made a choice. In panel c, hyperparasitoids experienced on the wheat-host complex are shown in yellow. The proportion of hyperparasitoids that did not respond to either odor source (no response) is presented in the right panels; $N$ is the total number of individual females tested per combination of hyperparasitoid and plant treatment. The number of individual plants per treatment combination was 5 in panel a, 10-17 in panel b and 8-10 in panel c. Per sub-experiment, significance of the effect of hyperparasitoid treatment is indicated on the right side of each set of bars (GLM). Using the intercept-only models, the $P$-value of the intercept was then used to determine whether hyperparasitoid choice deviated significantly from a 50:50 distribution (indicated in the green bars because pepper-experienced hyperparasitoids were used as the reference level, $P>0.05$, ns) $\chi_{1}^{2}=1.85, P=0.17$, respectively), nor on the number of mummies present on the mummy-infested plant (GLM, $\chi^{2}{ }_{1}=3.25, P=0.071$ and $\chi_{1}^{2}=1.98, P=0.16$, respectively; see supplementary Fig. S1).

To verify if our results were specific to the plant species we used, we performed an additional experiment with mummy-infested wheat plants infested with A. colemani mummies developed on $R$. padi aphids (Fig. 2c and supplementary Fig. S1). Again, hyperparasitoid females did not differentiate between the volatiles from mummyinfested plants when tested against uninfested wheat plants (GLM, $z=0.76, P=0.45$ ), independent of prior exposure to the host-complex on wheat or pepper (GLM, $\chi_{2}^{2}=1.87$,
$P=0.39)$ or the number of mummies on infested plants $\left(\chi^{2}{ }_{1}=0.48, P=0.49\right)$.

\section{Experiment 3: attractiveness of mummy volatiles in the absence or presence of plant volatiles}

In the first two experiments, we could not disentangle the effect of plant and host volatiles on attraction of D. aphidum because the entire plant-host complex was used as an odor source. Therefore, we tested the response of D. aphi$d u m$ to volatiles from the host separately, either or not in combination with plant volatiles. Volatiles from 100 aphid mummies (A. colemani developed on $M$. persicae) were 
highly attractive to naïve $D$. aphidum females when tested against clean air (GLM, $z=2.64, P=0.008$; Fig. 3). However, attractiveness of mummy volatiles in combination with volatiles from an uninfested sweet pepper plant was significantly reduced (GLM, $\chi^{2}{ }_{1}=6.65, P=0.01$ ). In addition, the proportion of hyperparasitoid females that did not make a choice doubled in a background of plant volatiles (GLM, $\left.\chi_{1}^{2}=14.37, P<0.001\right)$.

\section{Discussion}

Our experiments have provided new insight into the foraging behavior of the aphid hyperparasitoid Dendrocerus aphidum. Although infochemicals derived from the plant-host complex were highly attractive when presented in the absence of other volatile odour sources, we found that $D$. aphidum has no preference for these volatiles when offered in a natural background of plant volatiles. Similarly, volatiles derived from the host were highly attractive to D. aphidum, but only in the absence of plant volatiles. This may suggest that plant volatiles have a different role in the foraging behavior of D. aphidum compared to previously studied hyperparasitoids that are associated with caterpillars and parasitoids of herbivorous hosts (Poelman et al. 2012). We think that this may be explained by the interaction between the hyperparasitoid's host stage and the plant (i.e. mummies that do not actively feed on the plant versus parasitized caterpillars that do) and the level of specialization of hyperparasitoids. More studies on hyperparasitoid responses to (induced) plant volatiles, on a diversity of four-trophic systems, are required to evaluate this hypothesis.

Like parasitoids of herbivorous arthropods, it has been suggested that hyperparasitoids may also use plant volatiles to locate their parasitoid host because these infochemicals are more detectable than volatiles from their small hosts (Poelman and Kos 2016). Plant volatiles may reliably indicate the presence of parasitoid hosts when parasitized herbivores induce the emission of specific volatiles, as shown for Lysibia nana (Poelman et al. 2012). This specialist hyperparasitoid uses the parasitoid Cotesia glomerata as its host, which is specialized on caterpillars that have a narrow host plant range. In contrast, our experiments provide little support for a role of HIPVs in host searching behavior of the aphid hyperparasitoid $D$. aphidum. Volatiles from the entire plant-host complex were highly attractive to naïve and experienced $D$. aphidum females but, because attractive mummy volatiles were also present, we cannot conclude that this response is due to HIPVs. Interestingly, we noticed a substantial reduction in the proportion of $D$. aphidum females that made a choice in an additional experiment where the insects were removed from the mummy-infested plants just prior to testing against uninfested pepper (66\% compared to $40 \%$, supplementary Fig. S2). This suggests that the presence of aphids and/or mummies on the plants indeed influenced the olfactory response of $D$. aphidum. On the other hand, we can also not conclude that $D$. aphidum does not respond to HIPVs at all. Volatiles from aphid-infested pepper plants on which mummies were not present still attracted D. aphidum, particularly females that were already experienced with host mummies, and particularly when aphids were parasitized. Parasitism of aphids may affect the plant's defense response (Vaello et al. 2018) but, to our knowledge, it is not known whether healthy and parasitized aphids induce different blends of HIPVs. Again, it is also possible that the presence of volatiles from the (parasitized) aphids themselves or volatiles from honeydew deposited on the plants may explain our findings. The congeneric aphid hyperparasitoid D. carpenteri is arrested by infochemicals from honeydew at short range (Buitenhuis et al. 2004), but it is not known

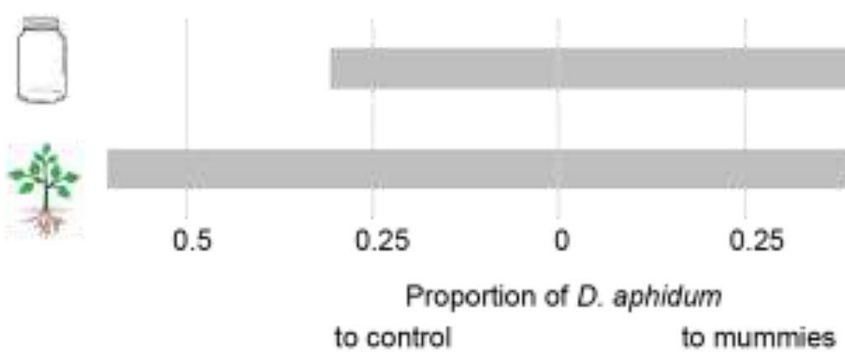

Fig. 3 Response of naïve D. aphidum to volatiles from their host in the absence and presence of plant volatiles in a Y-tube olfactometer. Hyperparasitoids were first presented with volatiles from $100 \mathrm{~A}$. colemani mummies developed on $M$. persicae aphids and clean air, and then to volatiles from the same mummies in a background of volatiles from uninfested sweet pepper plants. Bars represent proportions of hyperparasitoids that were attracted to the treatment (mummies) or control odor sources out of the number of hyperparasitoids that made a choice. The proportion of hyperparasitoids that did not respond to either odor source (no response) is presented in the right panels; $N$ is the total number of individual females tested over 6 experimental days. A GLM was used to evaluate the effect of background of plant volatiles on hyperparasitoid choice and to assess attractiveness of mummies based on the P-value of the intercept (using the experiment with mummy volatiles only as the reference level; $P<0.01$, **) 
whether honeydew volatiles are attractive over a longer distance. Further experiments are clearly needed to reveal if parasitized or mummified aphids can induce the emission of specific plant volatiles and whether these volatiles play a role in foraging behavior of D. aphidum. Based on the results of our other experiments and the biology of aphid mummy hyperparasitoids (see below), we expect that this role for $D$. aphidum and related species is limited at most.

Importantly, volatiles from the intact plant-host complex were not attractive to $D$. aphidum when tested in dual-choice experiments with plant volatiles as an alternative odor source instead of against clean air. Initially, we thought that this lack of preference may be due to low numbers of mummies on the infested plants. Low mummy numbers could be associated with reduced induction of HIPVs as it is known that aphid density influences HIPV emission (Turlings et al. 1998; Ponzio et al. 2017; Kroes et al. 2017), or could result in concentrations of attractive mummy volatiles below the detection limit of $D$. aphidum. However, mummy numbers did not significantly affect the olfactory behavior of D. aphidum in any of the experiments (supplementary Fig. S1). Moreover, in the sub-experiment with mummyinfested wheat plants, the numbers of mummies were substantially higher (mean \pm SE: $187 \pm 31, N=18$ ) than in any of the experiments with mummy-infested pepper plants, yet D. aphidum did not prefer volatiles from mummy-infested over uninfested wheat plants. We hypothesized that $D$. aphidum might have a stronger response to volatiles from the wheat-host complex because this system resembles the food-web in which $D$. aphidum evolved more closely (Fergusson 1980) than the pepper-host complex we used in the other experiments. Together, these results support the findings of Buitenhuis et al. (2005), who did not find attraction of four species of aphid hyperparasitoids to volatiles from host-infested potato plants when tested against volatiles from uninfested plants.

The effect of prior experience on olfactory preference of $D$. aphidum varied between experiments. We included a comparison between naïve and experienced hyperparasitoids because in parasitoid wasps a host-oviposition in the presence of HIPVs can lead to associative memory, resulting in an increased preference for these volatiles (Vet et al. 1990; Kruidhof et al. 2019; Little et al. 2019). In our experiments, experienced $D$. aphidum females were exposed to (induced) volatiles of plants and hosts and given the opportunity to oviposit in one or more mummies. Although it is not yet known whether learning occurs in hyperparasitoids, we hypothesized that this treatment would increase attraction of $D$. aphidum females to plant volatiles. In the first series of experiments, experienced $D$. aphidum females were more strongly attracted to volatiles than naïve ones but this depended on the particular odor source offered, with the effect being significant only in the choice situation between pepper plants infested with parasitized aphids and clean air. There was no effect of learning in the second experiment, with volatiles from mummy-infested plants tested against another plant odor source. Based on our experiments and findings, we cannot conclude if $D$. aphidum can learn to associate volatiles with the presence of their host or whether an oviposition experience increases motivation of D. aphidum to search for hosts without changing their olfactory preference. Dendrocerus aphidum and most other aphid mummy hyperparasitoids are generalists, and we predict that learning could play a role in local and/or temporal adaptation to available hosts.

Naïve $D$. aphidum females were strongly attracted to mummy volatiles. Mummies are required for reproduction and are indeed expected to emit reliable information to mummy hyperparasitoids. A previous study also reported attraction of the congeneric $D$. carpenteri to aphid mummies. Extracts from the mummy shell were also attractive and were shown to include several long-chain (C25-C33) alkanes, aldehydes and alcohols (Siri 1993). Although we did not test olfactory responses of $D$. aphidum to these compounds, it seems unlikely that they influence long-distance foraging behavior of Dendrocerus species, because their volatility is low. Attraction to host volatiles may be explained by the biology of hyperparasitoids of aphid mummies, such as D. aphidum. Dendrocerus species have a wide host range, using many different species of parasitoids, parasitizing many different aphid species that, in turn, may feed on a variety of plant species (Fergusson 1980; Sullivan and Völkl 1999). Being extreme generalists, Dendrocerus may have evolved innate olfactory responses to host volatiles that reliably indicate the presence of a variety of host species, rather than responses to (induced) plant volatiles. Nevertheless, individuals may be more specialized than the species as a whole. For example, the particular host in which they develop, may result in stronger olfactory responses to this type of mummy. Indeed, similar predictions have been made for generalist primary parasitoids because the great diversity of HIPVs that may be associated with host presence may constrain information processing (Vet and Dicke 1992). To further investigate whether $D$. aphidum responds innately to volatiles from its host, its olfactory responses to volatiles from mummies from different combinations of aphid and parasitoid species should be studied. Moreover, methods such as headspace entrainment should be used to investigate which infochemicals derived from aphid mummies may mediate their foraging behavior.

Surprisingly, mummy volatiles no longer attracted $D$. aphidum in the presence of volatiles from uninfested pepper plants. Additionally, when plant volatiles were added to mummy volatiles, the response rate of $D$. aphidum dropped significantly. Together with the findings of the first two experiments, this leads to two conclusions: (1) Plant 
volatiles appear to confuse rather than guide the foraging behavior of D. aphidum; and (2) Host volatiles are attractive to $D$. aphidum, but it seems unlikely that these infochemicals play a role in long-distance foraging behavior of D. aphidum in their natural environment. Instead, mummy volatiles may be important in host location after arrival of D. aphidum on a mummy-infested plant, as also suggested by previous studies (Siri 1993; Buitenhuis et al. 2005). The question of how D. aphidum females locate a host-infested plant from a distance remains unresolved. Although Sullivan and Völkl (1999) suggested over 20 years ago that hyperparasitoids of aphid mummies, such as Dendrocerus spp., may locate their hosts randomly rather than by using specific information, we now know that $D$. aphidum responds to certain infochemicals. In addition, a recent study showed that volatile blends emitted by specific bacteria may attract or repel D. aphidum females (Goelen et al. 2020), but it is not yet known how these microbial volatiles mediate the foraging behavior of hyperparasitoids in the field. Other possible mechanisms may include infochemicals from adult primary parasitoids, such as sex pheromones, or visual cues. However, D. carpenteri also forages at night, including dispersal flights between plants (Völkl and Kranz 1995), making the latter a less likely mechanism. Besides providing insight into the foraging behavior of fourth trophic level organisms, studies like ours and that of Goelen et al. (2020) may also contribute to developing management strategies for hyperparasitoids in a biological control context. Under some conditions, hyperparasitoids can kill nearly all parasitoids of aphids in greenhouse horticulture (Bloemhard et al. 2014), thereby representing a major constraint for the sustainable management of indoor-grown vegetables worldwide. Luring hyperparasitoids into traps with attractive baits has been suggested as a possible strategy to improve biocontrol (Cusumano et al. 2020). Our findings suggest that it may be challenging to develop such a strategy for $D$. aphidum because this species may not be able to locate an attractive trap in an environment with ample background (plant) volatiles, such as a commercial greenhouse. Nevertheless, the strong attraction of D. aphidum to volatiles from their mummy hosts (Fig. 3) or to microbial volatiles (Goelen et al. 2020) may still be exploited to lure hyperparasitoids into traps in empty greenhouses during crop rotation, allowing growers to start culturing a new crop in an environment that is free of hyperparasitoids.

Acknowledgements We appreciate the help of Thibault Costaz, Maartje van der Linden, Sabine Hoek and Aron Kuiper with experiments. This work was financed by the Netherlands Organisation for Scientific Research (NWO), domain TTW (project number 13848 granted to LEMV).

Author contributions All authors contributed to the study conception and design. Experiments were performed by $\mathrm{PJH}, \mathrm{DH}$, DJ and
PvS. Analyses were done by JGdB and results were interpreted by all authors. The first draft of the manuscript was written by JGdB and all authors commented on previous versions of the manuscript. All authors read and approved the final manuscript.

Funding This work was financed by the Netherlands Organisation for Scientific Research (NWO), domain TTW (project number 13848 granted to LEMV).

Data availability Data will be made publicly available in an appropriate repository.

Code availability Not applicable.

\section{Compliance with ethical standards}

Conflicts of interest The authors declare no conflicts of interest.

Ethics approval Not applicable.

Consent to participate Not applicable.

Open Access This article is licensed under a Creative Commons Attribution 4.0 International License, which permits use, sharing, adaptation, distribution and reproduction in any medium or format, as long as you give appropriate credit to the original author(s) and the source, provide a link to the Creative Commons licence, and indicate if changes were made. The images or other third party material in this article are included in the article's Creative Commons licence, unless indicated otherwise in a credit line to the material. If material is not included in the article's Creative Commons licence and your intended use is not permitted by statutory regulation or exceeds the permitted use, you will need to obtain permission directly from the copyright holder. To view a copy of this licence, visit http://creativecommons.org/licenses/by/4.0/.

\section{References}

Bloemhard CMJ, van der Wielen M, Messelink GJ (2014) Seasonal abundance of aphid hyperparasitoids in organic greenhouse crops in The Netherlands. IOBC-WPRS Bulletin 102:15-19

Buitenhuis R, McNeil JN, Boivin G, Brodeur J (2004) The role of honeydew in host searching of aphid hyperparasitoids. J Chem Ecol 30:273-285. https://doi.org/10.1023/b:joec.0000017977.39957.97

Buitenhuis R, Vet LEM, Boivin G, Brodeur J (2005) Foraging behaviour at the fourth trophic level: a comparative study of host location in aphid hyperparasitoids. Entomol Exp Appl 114:107-117. https://doi.org/10.1111/j.1570-7458.2005.00234.x

Cusumano A, Harvey JA, Bourne ME et al (2020) Exploiting chemical ecology to manage hyperparasitoids in biological control of arthropod pests. Pest Manag Sci 76:432-443. https://doi. org/10.1002/ps.5679

Cusumano A, Harvey JA, Dicke M, Poelman EH (2019) Hyperparasitoids exploit herbivore-induced plant volatiles during host location to assess host quality and non-host identity. Oecologia 189:699709. https://doi.org/10.1007/s00442-019-04352-w

de Boer JG, Salis L, Tollenaar W et al (2019) Effects of temperature and food source on reproduction and longevity of aphid hyperparasitoids of the genera Dendrocerus and Asaphes. Biocontrol 64:277-290. https://doi.org/10.1007/s10526-019-09934-4 
Dicke M, Van Loon JJA (2000) Multitrophic effects of herbivore-induced plant volatiles in an evolutionary context. Entomol Exp Appl 97:237-249. https://doi.org/10.104 6/j.1570-7458.2000.00736.x

Fatouros NE, Lucas-Barbosa D, Weldegergis BT et al (2012) Plant volatiles induced by herbivore egg deposition affect insects of different trophic levels. PLoS ONE 7:13. https://doi.org/10.1371/ journal.pone.0043607

Fergusson NDM (1980) A revision of the British species of Dendrocerus Ratzeburg (Hymenoptera: Ceraphronoidae) with a review of their biology of aphid hyperparasites. Bull Br Mus (Nat Hist) 41:255-314

Goelen T, Sobhy IS, Vanderaa C et al (2020) Volatiles of bacteria associated with parasitoid habitats elicit distinct olfactory responses in an aphid parasitoid and its hyperparasitoid. Funct Ecol 34:507520. https://doi.org/10.1111/1365-2435.13503

Grasswitz TR (1998) Contact kairomones mediating the foraging behavior of the aphid hyperparasitoid Alloxysta victrix (Westwood) (Hymenoptera: Charipidae). J Insect Behav 11:539-548. https://doi.org/10.1023/a:1022315413516

Kroes A, Weldegergis BT, Cappai F et al (2017) Terpenoid biosynthesis in Arabidopsis attacked by caterpillars and aphids: effects of aphid density on the attraction of a caterpillar parasitoid. Oecologia 185:699-712. https://doi.org/10.1007/s00442-017-3985-2

Kruidhof HM, Kostenko O, Smid HM, Vet LEM (2019) Integrating parasitoid olfactory conditioning in augmentative biological control: potential impact, possibilities, and challenges. Front Ecol Evol 7:84. https://doi.org/10.3389/fevo.2019.00084

Little CM, Chapman TW, Hillier NK (2019) Considerations for insect learning in integrated pest management. J Insect Sci 19:6. https:// doi.org/10.1093/jisesa/iez064

McCormick AC, Unsicker SB, Gershenzon J (2012) The specificity of herbivore-induced plant volatiles in attracting herbivore enemies. Trends Plant Sci 17:303-310

Poelman EH, Bruinsma M, Zhu F et al (2012) Hyperparasitoids use herbivore-induced plant volatiles to locate their parasitoid host. PLoS Biol 10:13. https://doi.org/10.1371/journal.pbio.1001435

Poelman EH, Kos M (2016) Complexity of plant volatile-mediated interactions beyond the third trophic level. In: Blande JD, Glinwood R (eds) Deciphering chemical language of plant communication. Springer International Publishing, Cham, pp 211-225

Ponzio C, Papazian S, Albrectsen BR et al (2017) Dual herbivore attack and herbivore density affect metabolic profiles of Brassica nigra leaves. Plant Cell Environ 40:1356-1367. https://doi.org/10.1111/ pce. 12926

Singh R, Srivastava PN (1987a) Potential host-habitat location by Alloxysta pleuralis (Cameron) (Alloxystidae: Hymenoptera). Zeitschrift für Angewandte Zoologie 74:337-341

Singh R, Srivastava PN (1987b) Factors associated with host location by Alloxysta pleuralis (Cameron), a hyperparasitoid of Trioxys indicus Subba Rao and Sharma (Alloxystidae: Hymenoptera/ Aphidiidae: Hymenoptera). Entomon 12:325-328

Siri N (1993) Analysis of host finding behaviour of two aphid hyperparasitoids (Hymenoptera: Alloxystidae, Megaspilidae). Ph.D. Thesis, Christian-Albrechts University
Sullivan DJ, Völkl W (1999) Hyperparasitism: multitrophic ecology and behavior. Annu Rev Entomol 44:291-315. https://doi. org/10.1146/annurev.ento.44.1.291

Turlings TCJ, Bernasconi M, Bertossa R et al (1998) The induction of volatile emissions in maize by three herbivore species with different feeding habits: possible consequences for their natural enemies. Biol Control 11:122-129. https://doi.org/10.1006/ bcon.1997.0591

Turlings TCJ, Erb M (2018) Tritrophic interactions mediated by herbivore-induced plant volatiles: mechanisms, ecological relevance, and application potential. Annu Rev Entomol 63:433-452. https ://doi.org/10.1146/annurev-ento-020117-043507

Vaello T, Sarde SJ, Marcos-Garcia MA, et al (2018) Modulation of plant-mediated interactions between herbivores of different feeding guilds: Effects of parasitism and belowground interactions. Sci Rep 8:. https://doi.org/10.1038/s41598-018-32131-9

van Neerbos FAC, De Boer JG, Salis L et al (2020) Honeydew composition and its effect on life-history parameters of hyperparasitoids. Ecol Entomol 45:278-289. https://doi.org/10.1111/een.12799

Vet LEM (1999) Evolutionary aspects of plant-carnivore interactions. In: Chadwick DJ, Goode J (eds) Insect-plant interactions and induced plant defence. Wiley, Sussex, pp 3-20

Vet LEM, Dicke M (1992) Ecology of infochemical use by natural enemies in a tritrophic context. Annu Rev Entomol 37:141-172. https://doi.org/10.1146/annurev.en.37.010192.001041

Vet LEM, Lewis WJ, Papaj DR, van Lenteren JC (1990) A variableresponse model for parasitoid foraging behavior. J Insect Behav 3:471-490. https://doi.org/10.1007/BF01052012

Vet LEM, Wackers FL, Dicke M (1991) How to hunt for hiding hoststhe reliability-detectability problem in foraging parasitoids. Neth J Zool 41:202-213

Völkl W, Kranz P (1995) Nocturnal activity and resource utilization in the aphid hyperparasitoid, Dendrocerus carpenteri. Ecol Entomol 20:293-297. https://doi.org/10.1111/j.1365-2311.1995.tb00459.x

Völkl W, Sullivan DJ (2000) Foraging behaviour, host plant and host location in the aphid hyperparasitoid Euneura augarus. Entomol Exp Appl 97:47-56. https://doi.org/10.104 6/j.1570-7458.2000.00715.x

Zhu F, Broekgaarden C, Weldegergis BT et al (2015) Parasitism overrides herbivore identity allowing hyperparasitoids to locate their parasitoid host by using herbivore-induced plant volatiles. Mol Ecol 24:2886-2899. https://doi.org/10.1111/mec.13164

Zhu F, Cusumano A, Bloem J et al (2018) Symbiotic polydnavirus and venom reveal parasitoid to its hyperparasitoids. Proc Natl Acad Sci USA 115:5205-5210. https://doi.org/10.1073/pnas.17179 04115

Zhu F, Weldegergis BT, Lhie B et al (2014) Body odors of parasitized caterpillars give away the presence of parasitoid larvae to their primary hyperparasitoid enemies. J Chem Ecol 40:986-995. https ://doi.org/10.1007/s10886-014-0500-7 